\title{
Article \\ Exploring the effects of Clustering Algorithms on Free Text Recommendation
}

\author{
Israel Mendonça ${ }^{1 *}$, Akira Fukuda ${ }^{1}$, Antoine Trouve ${ }^{2}$, and Kazuaki Murakami ${ }^{2}$ \\ 1 Kyushu University, 744-Motooka, Nishi-Ku, Fukuoka, Fukuoka, Japan; \\ 2 Team Aibod, 1-8-7-Daimyo, Chuo-Ku, Fukuoka, Fukuoka, Japan \\ * Correspondence: israel.m.san@gmail.com
}

\begin{abstract}
In this paper, we provide a study on the effects of applying classical clustering algorithms, such as k-Means to free text recommender systems. A typical recommender system may face problems when the number of items from a database goes from a few items to hundreds of items. Currently, one of the most prominent techniques to scale the database is applying clustering, however clustering may have a negative impact on the accuracy of the system when applied without taking into consideration the underlying items. In this work, we build a conceptual text recommender system and use k-Means to partition its search space into different groups. We study how the variation of the number of clusters affects its performance in the light of two performance measurements: recommendation time and precision. We also analyze if this clustering is affected by the representation of text we use. All the techniques used in this study uses word-embeddings to represent the document. One of the main findings of this work is that using clustering we can improve the recommendation time in up to almost 30 times without affecting much off its initial accuracy. Another interesting finding is that the increment of the number of clusters is not directly translated into linear performance.
\end{abstract}

Keywords: Clustering, Recommender-Systems, Word-Embedding

\section{Introduction}

Recommendation Systems are systems specialized in retrieving items from a database that may be of interest of users (top- $n$ recommendation problem). A typical Recommendation System will collect information from an user inputs and match this information with a set of heuristics to fetch the items it believes will best suit the user needs. Efficient recommendation systems can retrieve meaningful items in a short amount of item, i.e., the accuracy and the retrieval time of the system are widely used as metrics to evaluate recommendation systems.

The structure of the data directly influences the capacity of a system to analyze the items parameters and produce recommendations. However, natural text documents does not have a clear constitutional rule, and can vary greatly in terms of size, contents and internal text structure. Because of that, when one wants to create a recommendation system, she requires to apply techniques that are able to generate a structure that represents these free text documents. The most classical representation approaches are: Bag of words (BOW), in which each document is represented as the set of its internal words, with variations removing or not stopwords, and term-frequency inverse-document-frequency (tf-idf), in which each word in the document has its importance calculated by the frequency it appears in the text against the frequency it appears in the corpora.

Classical document representations often fails to capture the whole complexity that exists in the natural language text field, like synonyms or polysemous words (words with several meanings). Word2Vec models were introuced in 2013 by Mikolov, et. eal. [1] with the main objective of generating some structure to represent words that could carry their semantic meaning. In their technique, words are represented as a high-dimensional vector, that are generated from the probabilities of their existence in a corpora. From their work, different authors employed word-embedding to calculate the distance between documents. 
When we recommend documents by finding the closest documents in the search space, the amount of documents to compare directly influences the recommendation time. If we represent a document as a vector of $n$ dimensions and we have $d$ documents in our search space, the complexity of calculating the most similar items is defined as $\mathrm{O}(n \times d)$. However, if we reduce this search space by a fraction $k$ of its original value, the search time is also reduced by $\frac{n}{k}$. However, dividing the search space into $k$ fractions can hurt the accuracy of the system. For instance, the clustering algorithm could generate highly heterogeneous clusters, which could spread documents of the same classes among the clusters and reduce the probability of them being recommended when we provide recommendations from a single cluster.

In this paper, we apply a clustering technique to divide the search space of a recommendation system and calculate the influences on precision and recommendation time on this system. We also compare the effects of using clustering into different document representations. Both document representations uses word embeddings.

The rest of the paper is organized as follows. Section 2 presents a review of the literature regarding clustering and recommendation systems. Section 3 briefly explains some of the necessary background to better understand this work. Section 4 gives a more detailed explanation about the techniques we use in this paper. In Section 5, we introduce the datasets, the experimental setup, and the set of experiments conducted. In Section 6 we discuss the results of our experiments, we show that our methodology was able to improve the recommendation time by almost 4 times for our tested dataset. Finally, we conclude by highlighting the main points of our work, and identifying some possible perspectives.

\section{Related Works}

Cluster documents for reducing search time has been studied by different authors. On Cutting et. al. [2], and Hearst and Pedersen [3], clustering is used to build a table-of-contents of the documents collection and hence facilitate the browsing through the collection. Zamir and Etzioni [4] introduces a new clustering algorithm called the Suffix Tree Clustering (STC) which first identifies sets of documents that shares common phrases, and then clusters according to these phrases. Although we do not use the phrases for clustering, we do use the document's combined bag of word to generate the clusters.

Many authors uses content-based clustering for retrieving information from the web [5]. On [6] the author ranks the search query and then generate results based on this rank, they then merge these results to generate a cluster. We also use the contents of the document to cluster the documents, but instead of clustering when we return the document, we pre-cluster them based on their distances.

Although all of those techniques have efficient ideas of how to separate documents, none of them takes into consideration that the documents are a word-embedding form. To the best of our knowledge, we are the first authors to try to use classical clustering techniques to separate documents for a recommender system that uses word embeddings document representations.

\section{Background}

\subsection{Word2Vec}

Word2Vec is a word-embedding technique that was introduced by Mikolov et al. [1] and is based on the skip-gram model with sampling. They propose a simple neural network, with emphasis on efficiency at the training phase rather than precision. The neural network architecture consists of an input layer, a projection layer, and an output layer to predict nearby words. They train the vectors with the aim of maximizing the log probability of neighboring words within a specific corpus. More formally, given a sequence of words $w_{1}, \ldots, w_{T}$, it tries to maximize the average log probability.

$$
\frac{1}{T} \sum_{t=1}^{T} \sum_{-c \leq j \leq c, j \neq 0} \log p\left(w_{j}, w_{t}\right)
$$


where $c$ is the size of the training context and $p\left(w_{j} \mid w_{t}\right)$ is the hierarchical softmax function of the associated words $w_{j}$ and $w_{t}$. . Since the model is usually trained over large datasets, the words representation are able to learn complex word relationships and also carry semantic meanings. The learning phase of word embeddings is unsupervised and it can be computed on different corpus based on the user interest or pre-computed in advance. In this paper we use a pre-trained version of Google's word2vec [7] as our word embedder, but other embeddings techniques are also available (Collobert and Weston, 2008 [8]; Mnih and Hinton, 2009 [9] ; Turian et al., 2010 [10]).

\subsection{K-Means}

Clustering is the unsupervised classification of patterns into groups (clusters) [11]. The k-Means clustering is an iterative algorithm that initially assigns random groups to all the points, and then proceeds to its update step by computing the initial mean of the center each random assigned cluster. This update step will be run until the group's center doesn't variate much from each iteration. On termination the algorithm returns a list of each cluster it assigned to the data points.

More formally, given a set of dimensional training input vectors $x_{1}, x_{2}, \ldots, x_{n}$, the k-Means clustering algorithm partitions the $n$ training set into $k$ sets of data points or clusters $S=S_{1}, S_{2}, \ldots, S_{n}$, where $k \leq n$, such that the within cluster sum of squares is minimized. That is:

$$
\operatorname{argmin}(s) \sum_{i=1}^{k} \sum_{x \in S_{i}}\left\|x-c_{i}\right\|^{2}
$$

where $c_{i}$ is the centroid or mean of data point in cluster $S_{i}$. The algorithm tries to improve the result by updating the centroids described in Equation 2.

\section{Document Clustering for Recommendation Systems}

\subsection{A simple content-based recommendation system}

Figure 1 displays a general idea of a recommender system. In a content-based document recommendation system, when an input arrives, with respect to an input document, also called a query-document, a set of meta-data related to this query document is extracted and used by the system so it can, based on a set of internal beliefs, judge which items may be of the interest of the user. Then, the system needs to proceed to a search step in which it will look for its database for the items it intents to recommend. The recommendation can be divided into five distinct steps:

- Convert the document's contents to embedded vectors. This step converts every word of a document to its embedded version to be used by the document representation models.

- Convert the embedded vectors to a document representation. This step converts the set of word embeddings into a single vector. The shape of this vector depends on the representation used.

- Label from clustering algorithm. This step is responsible for getting the contents of the document and generating a label based on it. This label represents the group in which the document belongs.

- Documents retrieval. In this step, we select documents from the database to be recommended. Those documents will be used in the next ste.

- Sorting. In this step we apply a sorting algorithm to rank the documents based on their similarity to the query document.

Figure 2 displays the updated work-flow for our recommender system. In this section we describe each step in detail.

Once we have means to compare documents, and a function that can rank documents for recommendation, the last thing that the system requires is a tool to fetch items that will be passed along the query document to the rank function and produce a recommendation. On Subsections 4.4 and 4 we will introduce the techniques we use in this work to retrieve documents from the database. 


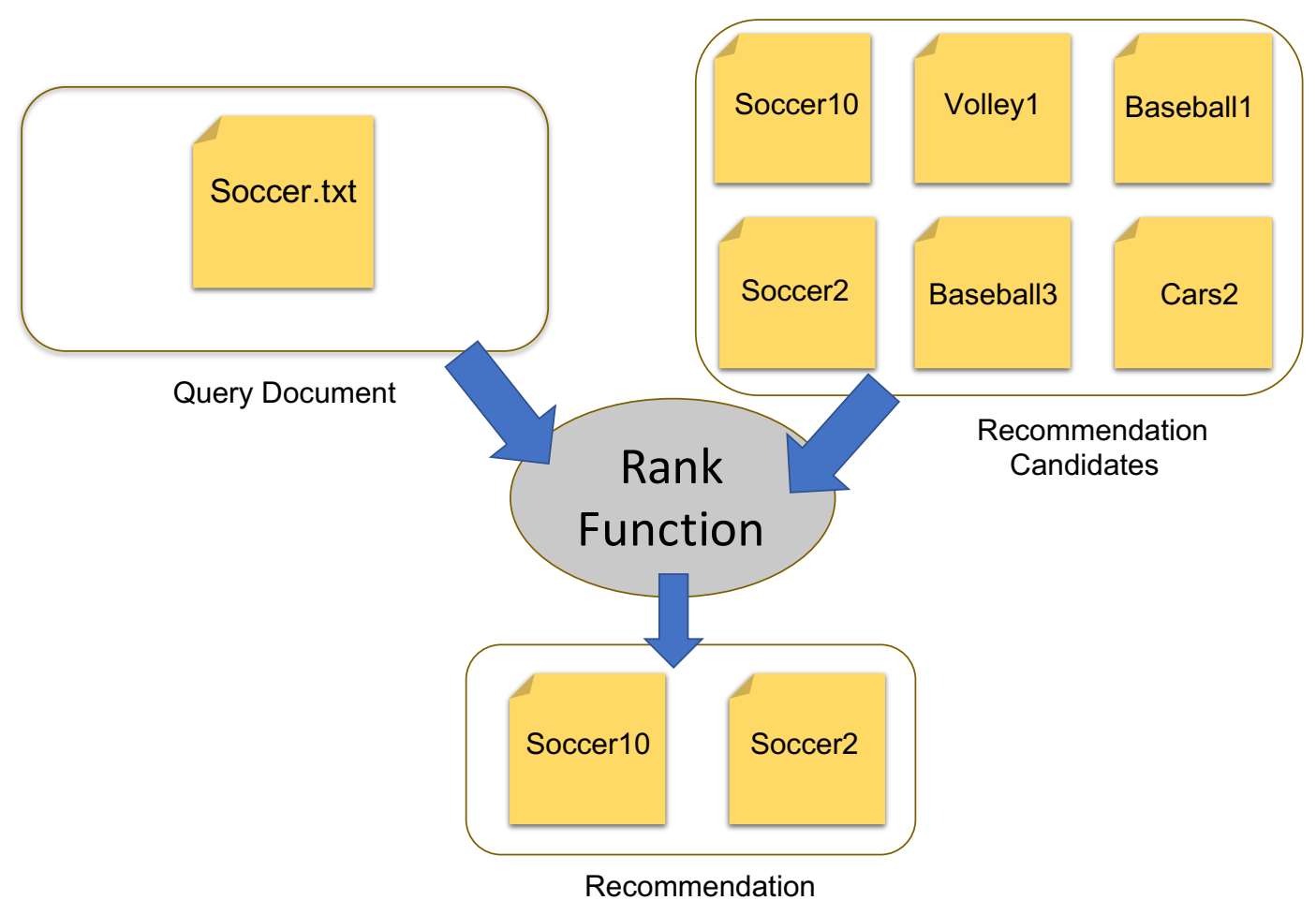

Figure 1. A simple recommendation: The system combines the query document and the database documents into a rank function to produce recommendations.

\section{Document to Word Embeddings}

In this step, we use a tokenizer to extract all the words of the document. Then we can use a word embedder to capture the semantic vector of each word. This step is identical for every document representation. At the end of this step, for each document, we obtain a set of keyed vector $v$ that represent the document.

\section{Generating Representations}

In this step, we would like to convert the document's keyed vectors $v$ into a single vector that contains the main meaning of the document.

Formally, for a matrix $M_{w, v}$ containing every word $w$ of a document in its vectorial form $v$. Let $X$ be the document representation converter function that applied on $M_{w, v}$ converts it into a vector $v_{x}$ that represents the document for this representation.

Since there is no straightforward mean of calculating the distance of two free-text documents, we had to generate a structure to represent documents, which provides us a tool to compare them. We use two document representation techniques, both uses word2vec to convert every document used in the system. The first technique is called Column Average Representation (CAR) and was introduced in [12] and uses the average of each column feature to represent documents. The second one is called Doc2vec and was introduced in [13] and uses concepts from word2vec to represent documents. Both techniques are explained in details on Subsections 4.2 and 4.3 respectively.

\section{Labeling a document}

So far, we have obtained the document representation for each document. Next we would like to cluster those documents based on their position in the search space using the methodology described in Section 4.5. We use K-Means to group all the documents contained in the database. Given the vectors of the documents of the database, we can generate a model that is able to not only separate the existing documents, but also capable of providing a cluster to new documents, in the form of a label. These labels will be used to the next step to retrieve documents from the database. 


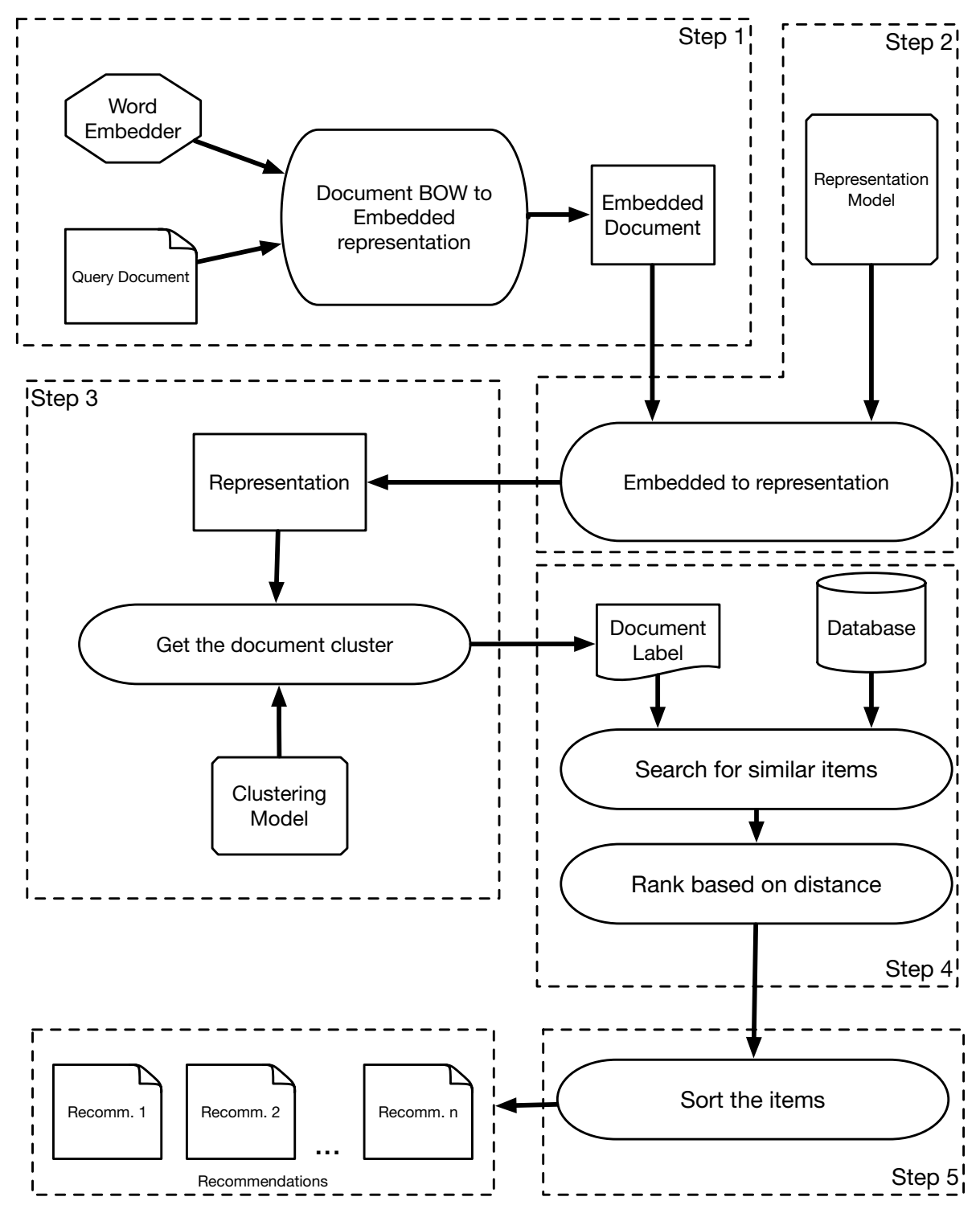

Figure 2. Recommendation Workflow: The first step converts the query document to an embedded version of it, the second step converts it to the representation, the third step searches for documents in the database and then, rank and sort them, finally the last step return the items sorted by rank.

More formally, for a document $d$ in its representation form $v_{x}$, let $y$ be the labeling algorithm that applied on $v_{x}$ generates a label $l$ to be used to the retrieval step to search documents.

\section{Document Retrieval}

In this step, we use the model generated by the previous step to selectively retrieve documents from the dataset. Every time we need to make a search in the database, we will select only members from a determined group of documents. This reduced amount of retrieved documents will reduce the load of the sorting algorithm since it will have less items to compare.

\section{Sorting and Ranking Documents}

This is the final step for recommending systems. In this step we rank the items according to their similarity with the query document. In this work, we will use a function based on distance to rank items and make recommendations. The closer a document from the recommendation space is from the query document, the higher will be its rank on the system. To calculate the distance we use the k-neighbors algorithm [14]. 

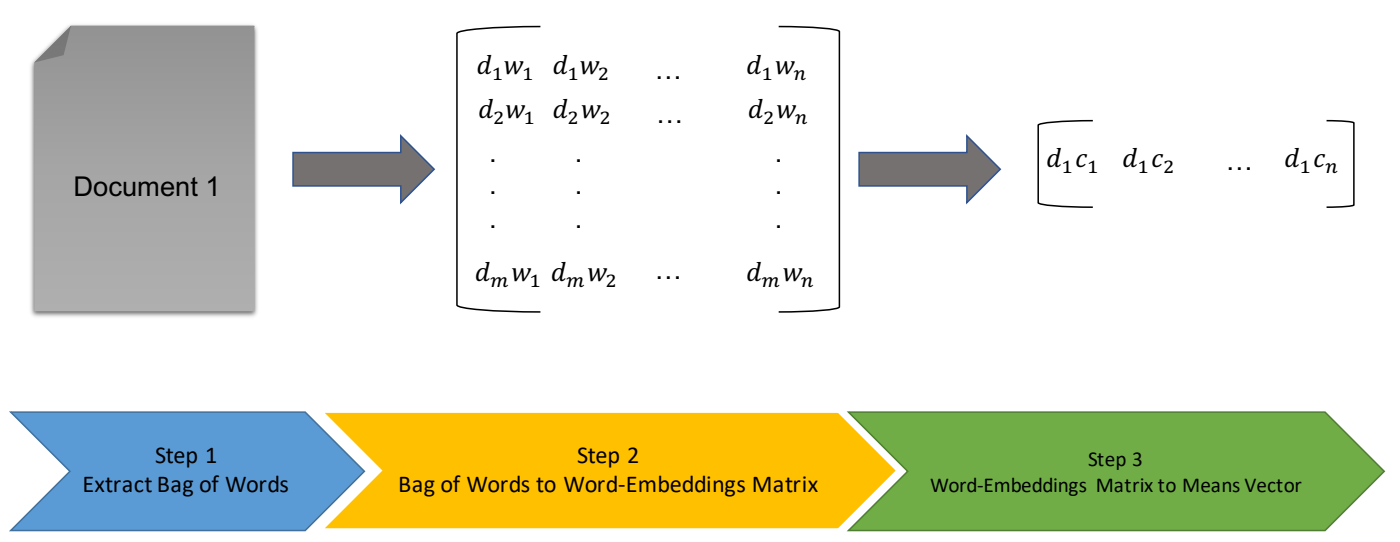

Figure 3. Document Representation: First we convert the document to a matrix of its bag-of-words, then we convert it to a single vector.

\subsection{Column Average Document Representation}

To be able to compare the distance of documents, we want to have a model that is able to move document representations from the textual domain to the numeric domain in order to be able to mathematically compare them.

The Column Average representation is a technique introduced in [12] that tries to grab the document meaning by extracting every word of the document, converting them to vectors, and averaging the vectors dimensions. By doing so, they represent documents as a vector and hence have a representation that allows us to directly compare documents.

The workflow of this method can be seen in Figure 3. The first step is to convert the document's text to the word embedding version. We use the word embedder to do such task. More formally, if we have a document $d$ containing a set of words $W$, we can convert every word of $W$ into embedded vectors and organize them into a matrix matrix $M_{i, j}$, in which each line $i$ represents a different word from the text and each column $j$ represents a feature of the word embedder. From this matrix we can calculate the mean of each column of $M$, which results into a vector $v$ with $j$ dimensions, in which each value $j$ of the vector is the mean of the corresponding column $j$ of $M$.

With this technique we are capable of converting free text documents to vectors that can be used in our recommender system. The length of each of those vectors depends only on the number of features of the word embedder, in the sense that every document vector has the same length, and therefore they can be directly compared, feature by feature.

A major advantage of using this technique when compared to other techniques is the complexity of the recommendation. A recommender system recommendation time is directly influenced by the size of the document representation, so a more compact representation becomes attractive for such system since having fast recommendations is always desired. By using the Column Average Representation, the complexity of the recommendation algorithm will be $O(n) \times D$, in which $D$ is the set of documents contained in the corpora.

\subsection{Doc2vec}

Doc2vec was presented in 2014 by Mikolov and Le [13] and tries to solve the problem of representing a document as a vector, regardless of the document length.

The concept they used was an extension of the word2vec model. They added another vector that contains the paragraph id, and maps it to a document.

They invented a model called Distributed Memory version of Paragraph Vector (PV-DM). It acts as a memory that remembers what is missing for the current context - or as the topic of the paragraph. While the word vectors represent the concept of a word, the document vector intends to represent the concept of a document. 


\subsection{Baseline Recommendation Characteristics}

As stated in Subsection 4.1, to be able to perform recommendations, the system needs to fetch documents into a database. Our baseline approach will fetch the whole database when making a recommendation.

To have an idea of the efficiency of this approach, we can calculate the complexity of the search on this set. Since we need to compare $n$ documents, represented as vectors $v$, the complexity of the baseline algorithm is $O(n \times v)$. Also, we need to perform a sorting operation after the distances are calculated. We use Timsort, which has complexity $O(n \times \log (n))$ for the average and worst case. So, the total complexity of a recommendation for our baseline case is $O(n \times v)+O(n \times \log (n))$.

\subsection{Our approach characteristics}

In our approach, we try to reduce the search time by clustering the results and searching on a fraction of the recommendation space.

In this work, we want to apply those clustering algorithms to our content-based recommender system in order to reduce the recommendation time when performing recommendations. The main motivation behind this idea is that as we separate the documents into groups, the amount of calculations required to make a recommendation are reduced. A reduction in the amount of calculations can be directly translated into a reduction in recommendation time. In order to test our hypothesis, we separate the whole database into clusters.

More formally, assume that for a query document $q_{i}$, contained in the test set $Q=q_{1}, \ldots, q_{n}$ the system returns a set containing a fixed number $k$ of documents determined $R=r_{1}, \ldots, r_{k}$. For each document of $R$, we will apply an arbitrary function $g$ that compares the class of the document $r_{k}$ and the query document $q_{i}$ and returns 1 in case they have the same class and 0 otherwise. Now assume that we have a search space that contains all the $d$ documents from the train set represented as one of the vectorial techniques introduced in the previous subsections.

If the documents are expressed in their vectorial form, we can represent them as a matrix $Z_{i, j} \in$ $\mathbb{R}^{d \times f}$. Each line of $Z$ contains a document $d$. The $j$-th column, $x \in \mathbb{R}^{f}$, represents the feature $f$ of the document in a multi-dimensional space. Every time the system receives a query document $q$, it will search for recommendation on its whole database. It will have to compare all the $f$ columns of each $d$ document and the $q$. The search algorithm performance will be $O(d \times f)$. If we partition this space $S$ and into $\beta$ sub-spaces $S S=s_{1}, \ldots, s_{\beta}, \beta \in S$, and perform searches on an arbitrary group $s_{\beta}$. It is expected that the number of comparisons are reduced $\beta$ times. Figure 4 illustrates how clustering can be used to prune the search space and improve recommendation time.

By dividing the search space, when we perform a search using a query document, we can use a model to, based on the query document contents, would select which group fits it better. If the groups are uniformly distributed, the expected speedup for the application is directly proportional to the number of clusters. Partitioning the search space means that we are able of reducing the complexity of searching for recommendation items from $\mathcal{O}(d \times c)$. to $\mathcal{O}\left(\frac{d \times c}{\beta}\right)$.

One of the main advantages of using clusters for recommender systems is that when the system searches for documents, there are multiple answers that can be viewed as correct. In our system, the evaluation is based on the class of the returned documents, which means that for the recommendation to be judged as useful, it only has to return documents from the same class of the query document. In this current of thought, the best partition would be the ones that evenly divide the documents into groups, and within each group there are only members of the same class. It is not incorrect for the system to have clusters containing members exclusively from a single class, in fact, the best case would be if the system separate the documents evenly among clusters. 


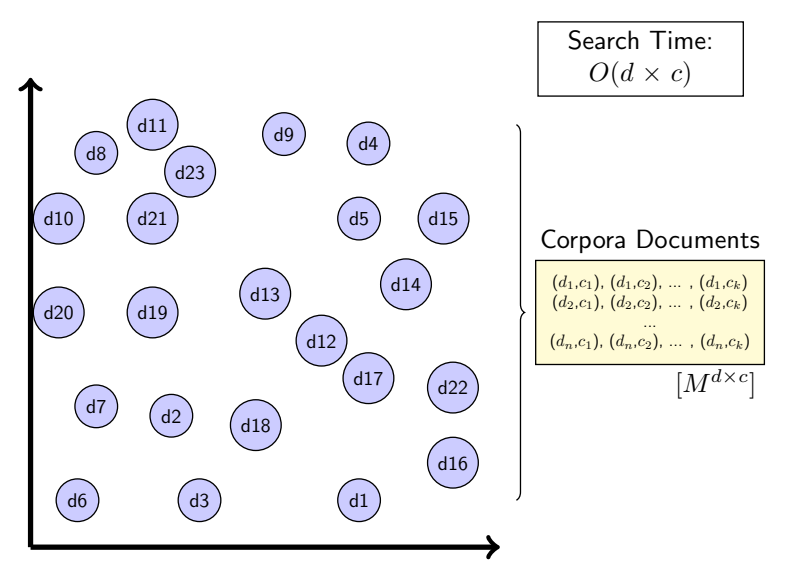

(a)

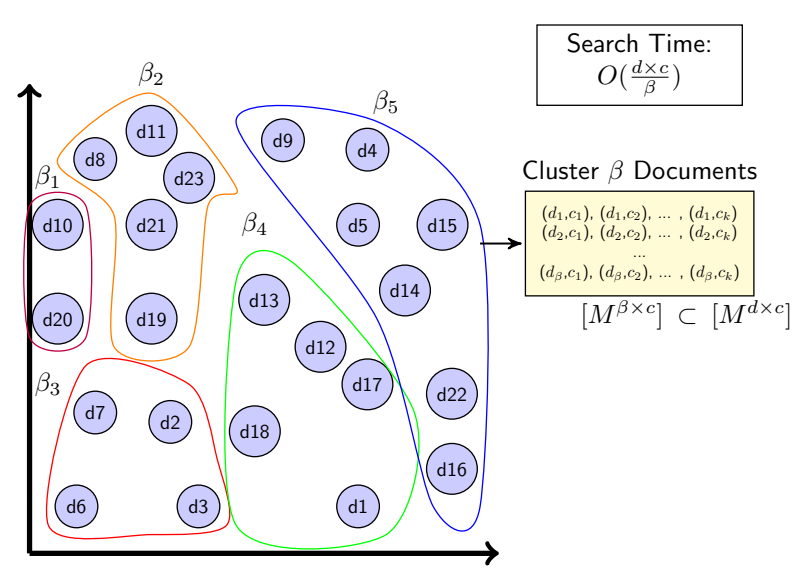

(b)

Figure 4. (a) Non-clustered corpora with search time of $O(d \times c)$. (b) Clustered corpora reducing the search time by a fraction $\beta$.

\section{Dataset and Experimental Setup}

This section will give details of the dataset we used as well as the experimental setup we used for our experiments.

\subsection{Dataset}

In the remainder of this article we use the Reuters-22172 [15] dataset to evaluate the performance of the recommendation system. Reuters is a classic news dataset labeled by news topics (we use the 8 -class version). It contains 8 classes, 5485 training documents and 2189 test documents. We will evaluate the quality of the recommendations based on whether or not the recommended documents are in the same class as the query document.

\subsection{Experimental Setup}

To test our model, we created a reference implementation of our method. We created a script that convert all the documents from a folder to the representation explained before and store them in a database. Then another script clusters those documents, and store the model that assign clusters to the file system. The information related to the cluster that each train document belongs is then stored into the database. Finally we run another script that takes the whole test set, convert each document to the representation introduced before and then calls a recommendation routine for each of them. The recommendation routine is responsible for reading the query document, and using the cluster model 
to assign a cluster to this document. It then retrieves all the documents from the assigned cluster from the database. It will then calculate the distance between each document and the query document, sort the results, and return the $k$ first documents.

To extract the bag-of-words of a document, we used the NLTK's sentence tokenizer and extracted all the words from sentences. For all the steps that required clustering, we used the sci-kit learn's version of K-Means. For the steps that required database for storing documents and labels, we used MongoDB.

Regarding word-embeddings, we download the pre-trained one from Google's webpage. To load the model and furthermore be able to convert words to its respective embeddings, we used Gensim, an open-source tool that can be used to load word pre-trained embedding and converts words to an embedding vector.

All the code necessary to run those experiments, and all the experimental results introduced on the next sections is public and available at github ${ }^{1}$.

Our experiments were run in a Ubuntu 17.10 virtual machine hosted in a dedicated 10-core 3.2GHZ Intel i7-6900K with $16 \mathrm{~Gb}$ of memory.

\section{Experimental Results}

In this section, we will present the experimental results for the system and dataset introduced in Section 5. In this recommender system, we will use both document representations introduced in Section 4, and we will analyze how the system will perform using each of them with and without clustering.

\subsection{Testing and Analysis Criteria}

The system in this work will be evaluated based on two metrics: recommendation time and precision. The recommendation time is the total time the system takes to perform all the recommendations. Precision is the ratio between the number of recommended documents that are relevant and irrelevant.

For the data described in Subsection 5.1, we recommend 10 documents for each document of the test set. This means that for our test set of 2189 documents, we will make 2189 recommendations, each of them returning up to 10 documents. We then compare the classes of the test document and recommended items to calculate the precision. The recommendation time will be calculated by summing the time of each individual recommendation.

\subsection{Time Efficiency}

We start our analysis by evaluating how much clustering influences recommendation time. Figure 5 displays the sum of each individual recommendation time for against the number of clusters. For our first case, the baseline in which all the documents are grouped into a single cluster, the total recommendation time is 795 seconds, which averages a time of 0.36 second per recommendation.

After the baseline, we increase the number of clusters from 8 to 104 (with steps of 8 ) and display their time. We notice that by using clustering only, regardless of the number of selected clusters, we have a positive impact over the recommendation time, as expected. We can also see that although the total recommendation time keeps decreasing from 8 clusters until we have 24 clusters, the recommendation time of the system starts to increase until we reach 48 clusters, after that it reduces to 258 seconds and stabilizes on numbers near 270 seconds for the rest of the experiment.

The system in general had an overall improvement, reaching highly improvements specially when we had over 100 clusters. Doc2Vec was the one that benefit the most from using clustering to

1 https://github.com/israelzinc/recommendation-clustering 


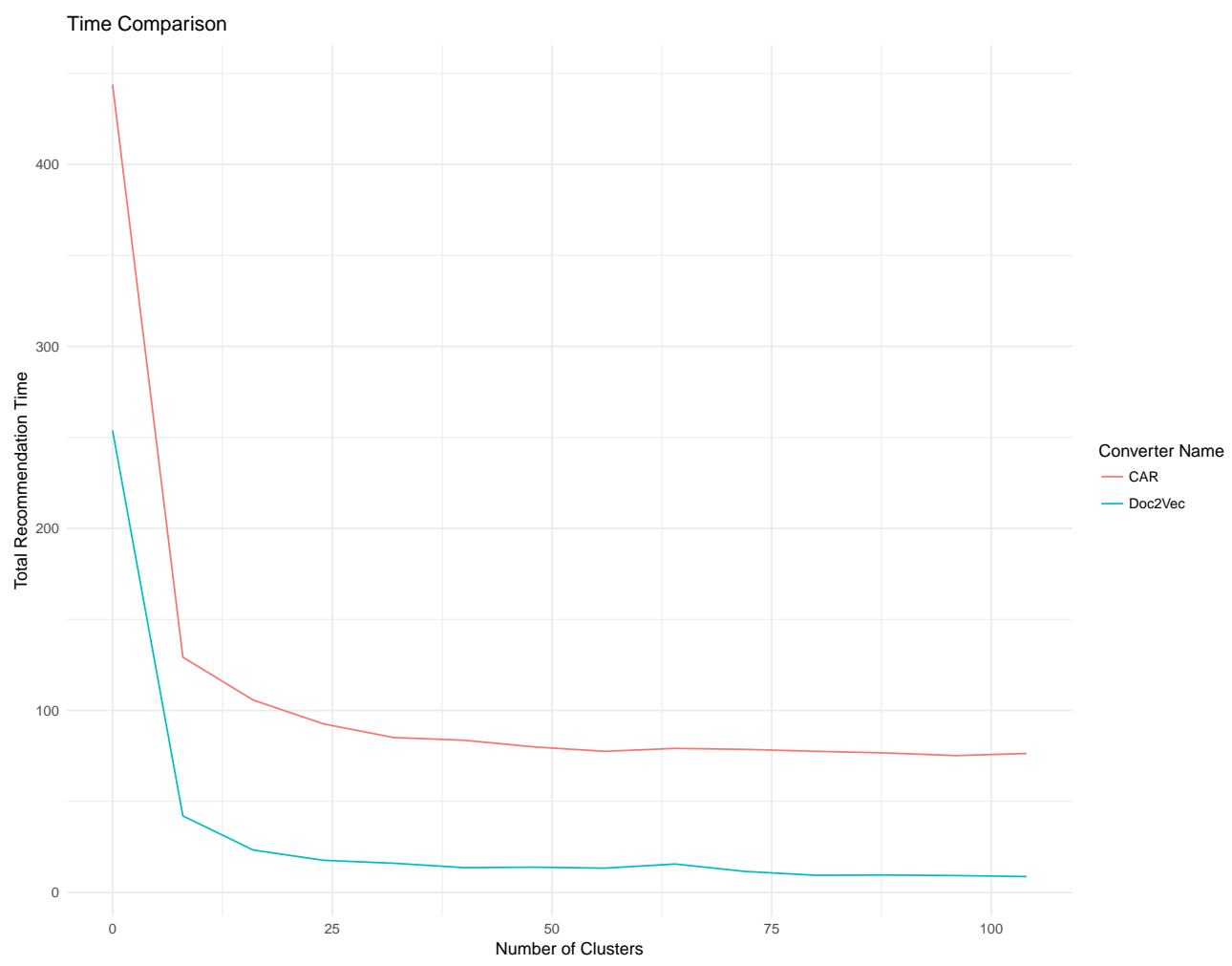

Figure 5. Reduction of the size of the document representation by means of using our model. The model is able to reduce the size of the corpora representation from a $3 \mathrm{D}$ matrix to a $2 \mathrm{D}$ one.

separate the database, having a speedup of near 30 times. Column Average Representation also had a significant improvement, being the most significant of them the one when we went from using no clustering (1 cluster) to using clusters. Table 1 gives us more details about the maximum variance on time for each representation.

Despite the system overall positive results using clustering, our maximum speedup was far from the theoretical speedup that could have been achieved. One of the reasons that may be causing this disparity between the real speedup and the theoretical one may be in the division of documents. If the clusters are separated evenly, this speedup should be linear, since the system would require $\frac{1}{n}$ less calculations to achieve a result. In order to better understand the effects of the partition configuration over the time, we take a closer look into the configuration of the partitions.

Table 1. Maximum difference in time and precision for each document representation.

\begin{tabular}{llcc}
\hline & Converter Name & Time Variance Speedup (x times) & Precision Variance (x times) \\
\hline 1 & baseline & 5.90 & 1.01 \\
2 & $\mathrm{~d} 2 \mathrm{v}$ & 29.03 & 1.03 \\
\hline
\end{tabular}

\subsection{Assessing the Cluster Partitions}

Figure 4 shows the distribution of members among the clusters for the following cluster sizes: 8 , 24,40 and 104 clusters. The ideal speedup is obtained when we have the same amount of documents inside each cluster, however as Figure 4 shows, the distribution of elements is not even among the clusters, which causes an imbalance on the recommendation time. Small clusters yield shorter execution times, while larger clusters have recommendation time closer to the baseline. In the end, the overall recommendation time is heavily influenced by the size of the clusters selected for the search stage. 


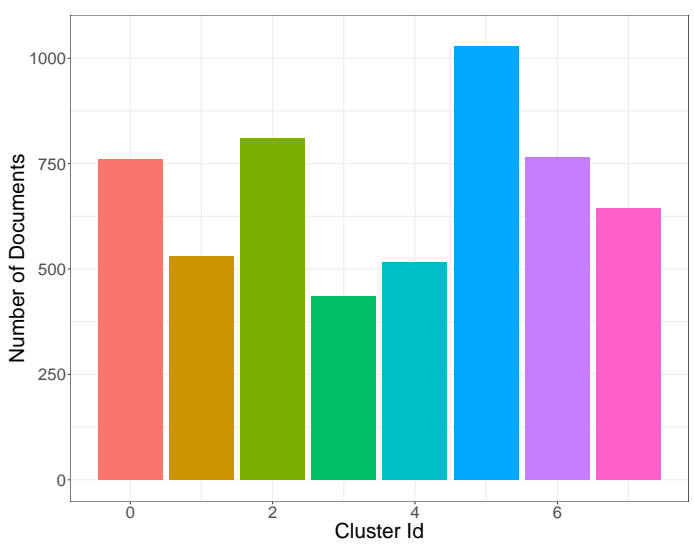

(a) 8 Clusters

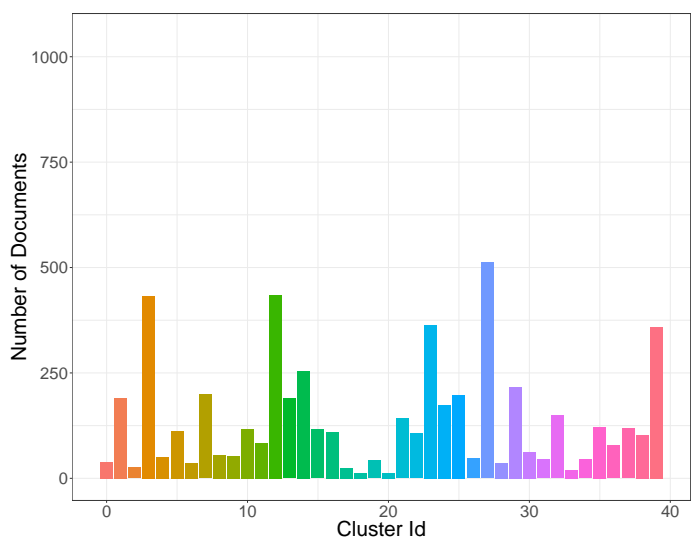

(c) 40 Clusters

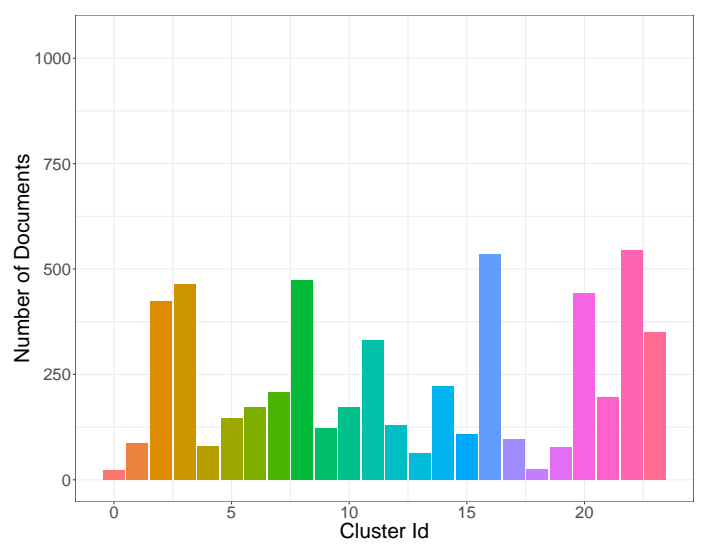

(b) 24 Clusters

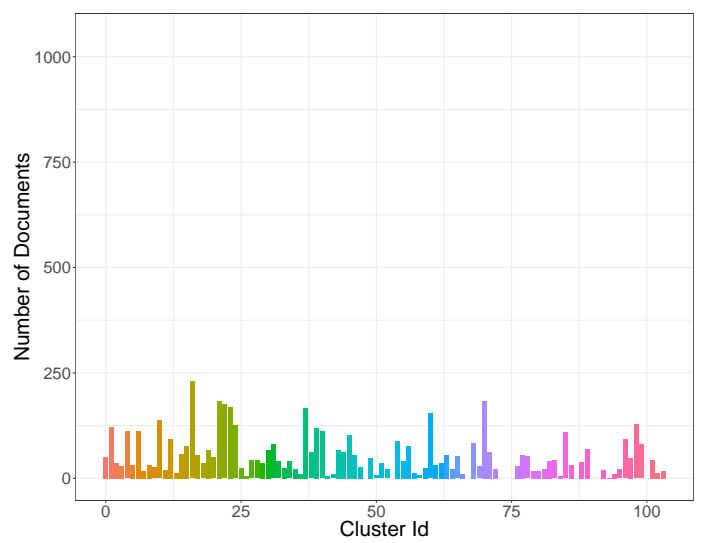

(d) 104 Clusters

Figure 6. Cluster individuals distribution for every cluster using k-Means with 8(a), 24(b), 40(c) and 104(d) clusters configuration. The higher the number of clusters, the more distributed the elements should be among the clusters.

To reason over the difference in time of a small and a big cluster, we analyze the recommendation time of each cluster size for two out of four cases aforementioned. Figures 7 and 8 displays the recommendation time separated by cluster size for each $\mathrm{k}-M e a n s$ version. The recommendation time grows as the number of elements inside a cluster grows. For 8 clusters, we can see that the bigger cluster has 2 times the size of the smallest one, but it can reach up to 4 times the recommendation time. For 24 clusters, this difference cannot be so easily perceived because the clusters have a more similar size, and consequently a more similar recommendation time.

\subsection{Effects on Precision}

We compute the precision by calculating the amount of correct guesses inside the set of recommended items. If we recommend 10 items, and 8 are from the same label as the query document, we say that the recommendation had $80 \%$ of precision.

Since we artificially separated the data, we expected the precision to be negatively impacted. However, the impact on the precision were minimal. The results for the baseline alongside with all the cluster sizes is displayed on Figure 9.

Regardless of the cluster size, the precision is not strongly affected. From the table we can draw two important results: The first one concerns the fact that the precision did not change significantly as the number of clusters changed, independently of the representation used. This result means that k-Means was able to take advantage of the document representation and generate highly accurate partitions. The second observation regards the document representations: From the baseline case 


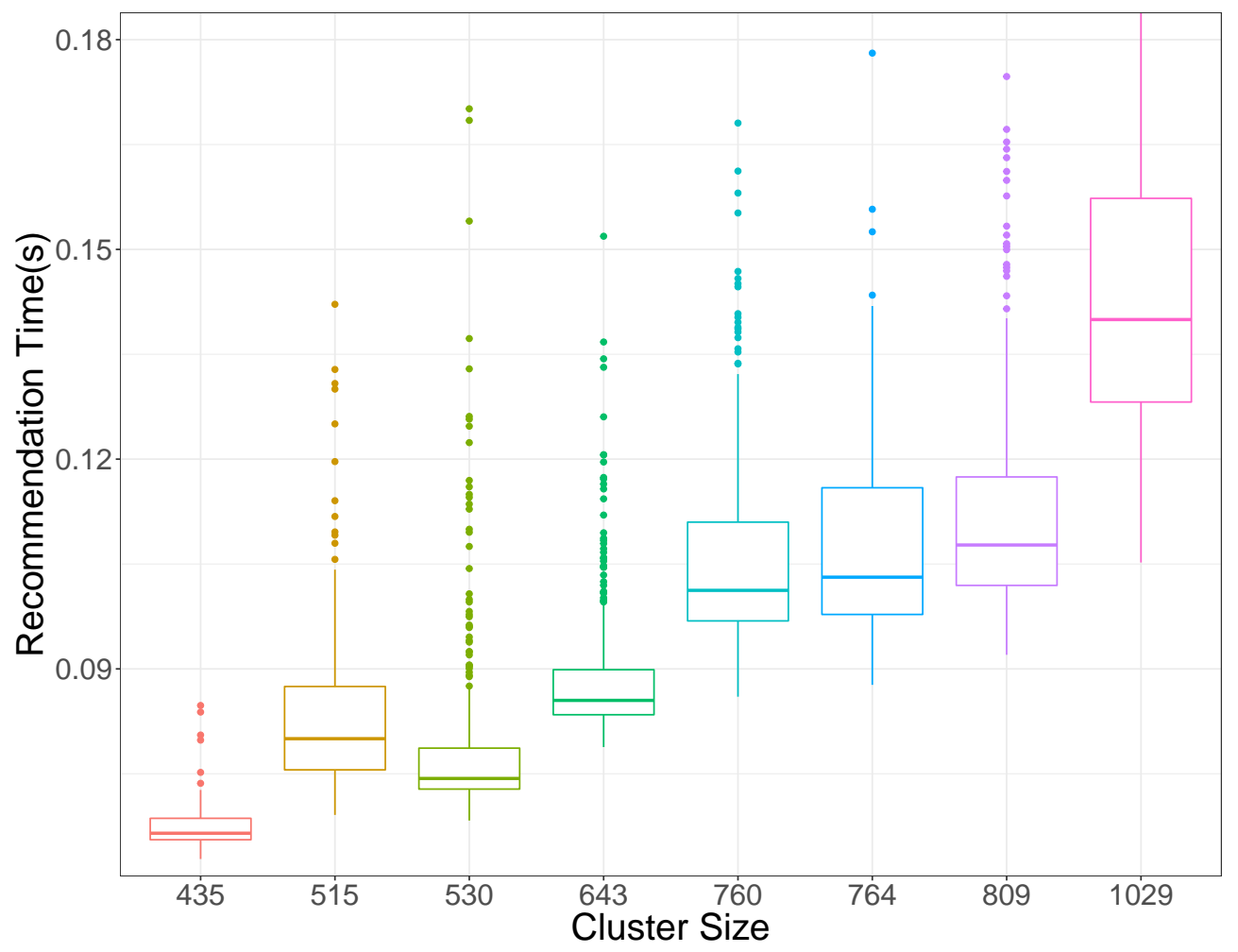

Figure 7. Recommendation time for every cluster size generated using k-Means with 8 clusters. We can see a pattern that the recommendation time increases as function of the cluster size.

without clustering, we had $90 \%$ precision for the CAR representation and around $55 \%$ for the Doc2vec representation. From these results we can draw the conclusions that both the document representations chosen for this experiment were sufficient accurate to capture the distinction that separates documents for this dataset; With CAR being more precise than Doc2vec. A more elaborated discussion over those two and other document representations can be found at [12].

\section{Conclusion and Future Works}

In this paper, we have studied the effects of adding clustering when doing recommendations of text files. Our main goal was to reduce the search time, without greatly affecting the precision. We investigated how different number of clusters for k-Means can affect the performance in relation of time and accuracy. We empirically tested our hypothesis using a real-world dataset and assessed that the time can be improved up to almost 30 times when using a correct configuration of clustering. We also verified that disregarding the number of clusters or document representation, using k-Means to partition the documents usually yields improvements in terms of time, without affecting much of the precision.

Author Contributions: Conceptualization, M.I. and T.A.; Methodology, M.I. and T.A.; Software: M.I.; Investigation, M.I. and T.A; Writing-Original Draft Preparation, M.I.; Writing-Review \& Editing, M.I. and T.A.; Supervision, F.A.; Funding Acquisition, F.A. and M.K.

Funding: This research was funded by National Council of Scientific and Technological Development (CNPQ).

Acknowledgments: The authors are grateful to the National Council of Scientific and Technological Development (CNPQ) for its financial support through the Science Without Borders program, see (http:// /www.cienciasemfronteiras.gov.br).

Conflicts of Interest: The authors declare no conflict of interest. 


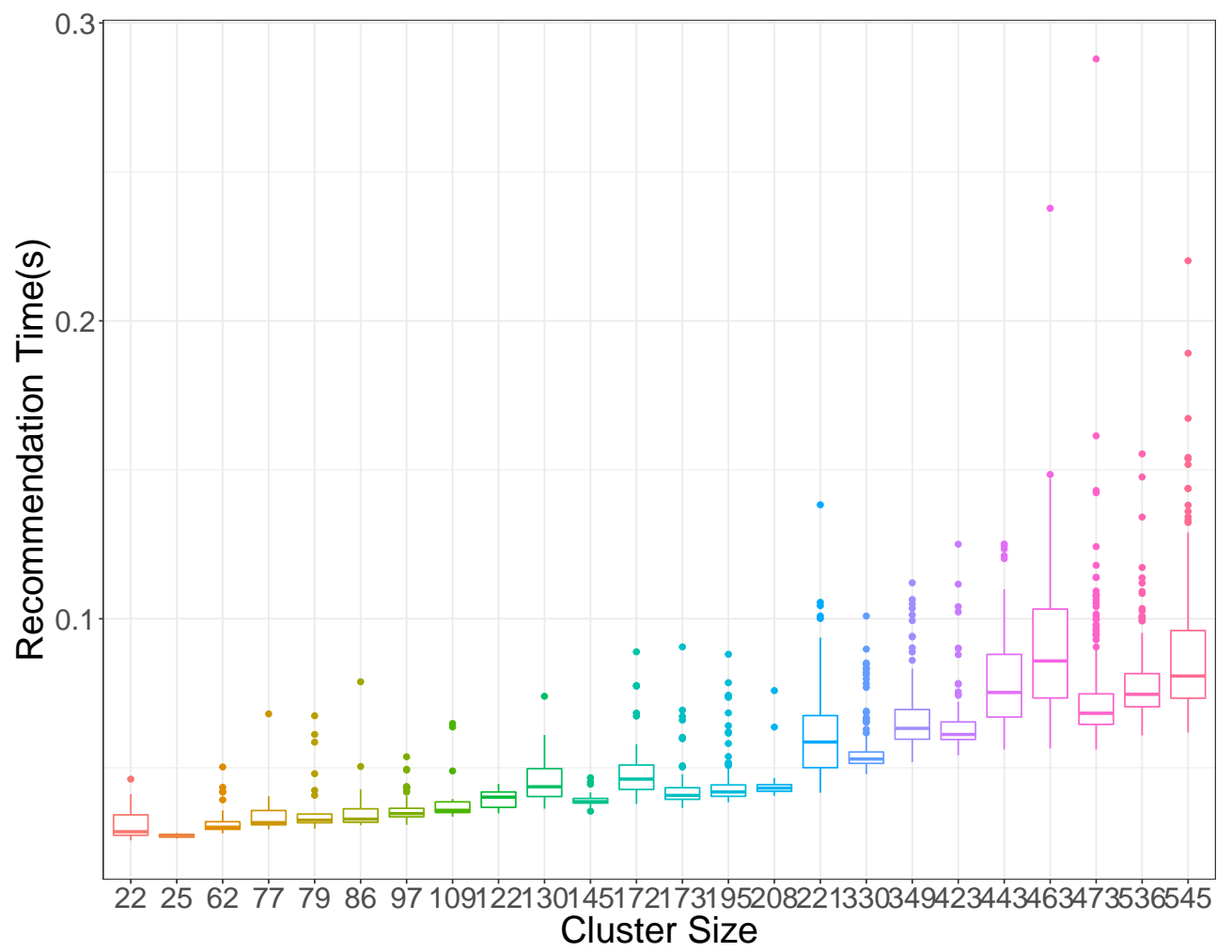

Figure 8. Recommendation time for every cluster size generated using k-Means with 24 clusters. We can see a pattern that the recommendation time increases as function of the cluster size.

\section{References}

1. Mikolov, T.; Chen, K.; Corrado, G.; Dean, J. Efficient estimation of word representations in vector space. arXiv preprint arXiv:1301.3781 2013.

2. Cutting, D.R.; Karger, D.R.; Pedersen, J.O. Constant Interaction-time Scatter/Gather Browsing of Very Large Document Collections. Proceedings of the 16th Annual International ACM SIGIR Conference on Research and Development in Information Retrieval; ACM: New York, NY, USA, 1993; SIGIR '93, pp. 126-134. doi:10.1145/160688.160706.

3. Hearst, M.A.; Pedersen, J.O. Reexamining the cluster hypothesis: scatter/gather on retrieval results. Proceedings of the 19th annual international ACM SIGIR conference on Research and development in information retrieval. ACM, 1996, pp. 76-84.

4. Zamir, O.; Etzioni, O. Web document clustering: A feasibility demonstration. Proceedings of the 21st annual international ACM SIGIR conference on Research and development in information retrieval. ACM, 1998, pp. 46-54.

5. Kobayashi, M.; Takeda, K. Information retrieval on the web. ACM Computing Surveys (CSUR) 2000, 32, 144-173.

6. Zeng, H.; He, Q.; Chen, Z.; Ma, W.; Ma, J. Learning to cluster web search results. SIGIR 2004: Proceedings of the 27th Annual International ACM SIGIR Conference on Research and Development in Information Retrieval, Sheffield, UK, July 25-29, 2004, 2004, pp. 210-217. doi:10.1145/1008992.1009030.

7. inc., G. word2vec, 2013. https://code.google.com/archive/p/word2vec/.

8. Collobert, R.; Weston, J.; Bottou, L.; Karlen, M.; Kavukcuoglu, K.; Kuksa, P. Natural language processing (almost) from scratch. Journal of Machine Learning Research 2011, 12, 2493-2537.

9. Mnih, A.; Hinton, G.E. A scalable hierarchical distributed language model. Advances in neural information processing systems, 2009, pp. 1081-1088. 


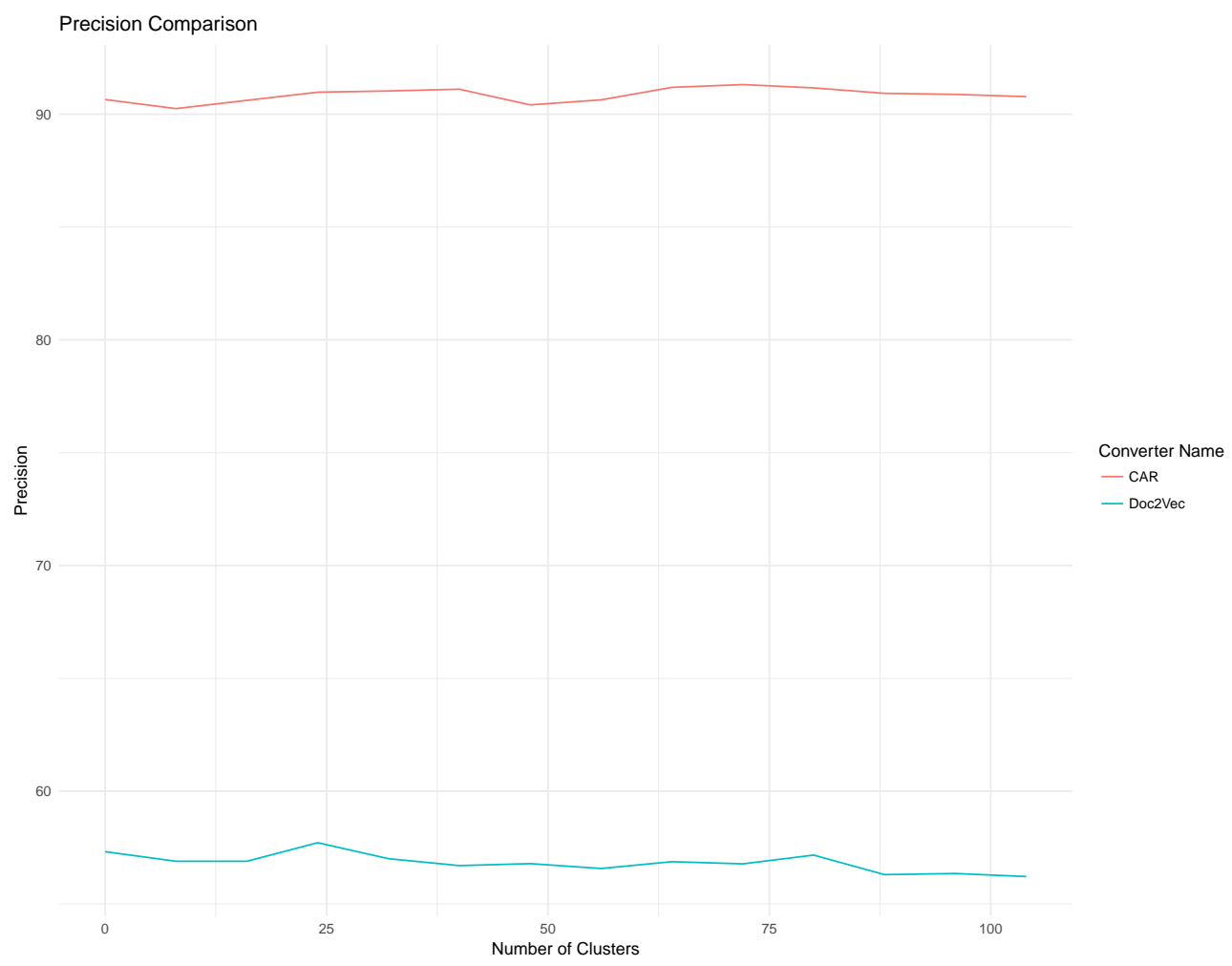

Figure 9. Precision Comparison for both document representations.

10. Turian, J.; Ratinov, L.; Bengio, Y. Word representations: a simple and general method for semi-supervised learning. Proceedings of the 48th annual meeting of the association for computational linguistics. Association for Computational Linguistics, 2010, pp. 384-394.

11. Jain, A.K.; Murty, M.N.; Flynn, P.J. Data clustering: a review. ACM computing surveys (CSUR) 1999, 31, 264-323.

12. Mendonca, I.; Trouve, A.; Fukuda, A.; Murakami, K. Exploring a Topical Representation of Documents for Recommendation Systems. The 9th IEEE International Conference on Awareness Science and Technology (iCAST 2018), 2018, pp. 95-100.

13. Le, Q.; Mikolov, T. Distributed representations of sentences and documents. International Conference on Machine Learning, 2014, pp. 1188-1196.

14. Altman, N.S. An introduction to kernel and nearest-neighbor nonparametric regression. The American Statistician 1992, 46, 175-185.

15. Lewis, D. Reuters-21578, 1997. https://archive.ics.uci.edu/ml/datasets/Reuters-21578+Text+Categorization+Collection. 